\title{
Evaluation of the fundamental properties of quantum dot infrared detectors
}

\author{
Jamie Phillips ${ }^{\text {a) }}$ \\ Department of Electrical Engineering and Computer Science, Solid State Electronics Laboratory, \\ The University of Michigan, Ann Arbor, Michigan 48109-2122
}

(Received 12 October 2001; accepted for publication 4 January 2002)

\begin{abstract}
The physical properties of detectors based on intraband optical absorption in quantum dots is described and examined in the interest of providing a competitive alternative infrared (IR) detector technology. These quantum dot detectors are an extension of quantum well infrared photodetectors and are expected to have a large performance advantage. A model is developed for quantum dot infrared photodetectors based on fundamental performance limitations enabling a direct comparison between IR materials technologies. A comparison is made among $\mathrm{HgCdTe}$, quantum well, and quantum dot IR detectors, where quantum dots are expected to have the potential to outperform quantum wells by several orders of magnitude and compete with $\mathrm{HgCdTe}$. In this analysis, quantum dots are expected to possess the fundamental ability to achieve the highest IR detector performance if quantum dot arrays with high size uniformity and optimal bandstructure may be achieved.

(C) 2002 American Institute of Physics. [DOI: 10.1063/1.1455130]
\end{abstract}

\section{INTRODUCTION}

Infrared (IR) detectors are important for a variety of applications, ranging from strategic and tactical defense applications to commercial applications in astronomy, public safety, and industry. High performance IR systems desire detectors with the highest performance, operation at the highest temperature, and fabrication at the lowest cost. The high performance IR detector technology leader of today is HgCdTe. ${ }^{1-5}$ However, this II-VI semiconductor material has difficulties in epitaxial growth and processing resulting in low yields, difficulties in achieving a viable production technology, and ultimately high cost. Because of these difficulties, alternative technologies have been investigated in III-V semiconductor materials. One of these technologies is the strained layer superlattice with a type-II band lineup ${ }^{6-8}$ (e.g., InAs/GaSb), which is expected to have reduced Auger scattering rates that may lead to increased operating temperatures in comparison to $\mathrm{HgCdTe}$. This promising technology is still in its infancy and requires complex material heterostructures. While this technology is an important developing technology, it will not be addressed here. Another alternative is the quantum well infrared photodetector (QWIP) ${ }^{9}$ which uses intersubband optical transitions in quantum wells as the detection mechanism. QWIPs have shown much success as demonstrated by their use in IR camera systems. ${ }^{10}$ However, QWIPs have shown much lower quantum efficiency than $\mathrm{HgCdTe}$ and require much lower operating temperatures to achieve the same level of performance of HgCdTe. An extension of QWIPs is the quantum dot infrared photodetector (QDIP), which uses optical transitions between bound states in the conduction/valence band in quantum dots. QDIPs are predicted to outperform QWIPs due to their inherent sensitivity to normal incidence radiation and reduced phonon scattering. QDIPs have been demonstrated by several

\footnotetext{
${ }^{a)}$ Electronic mail: jphilli@engin.umich.edu
}

groups ${ }^{11-17}$ where self-assembled quantum dots ${ }^{18-20}$ are fabricated using the Stranski-Krastanow growth mode during molecular beam epitaxy. These detectors have shown promising results, but are far from rivaling the current state of the art $\mathrm{HgCdTe}$ detectors. This article investigates the potential of QDIPs as a competitor to $\mathrm{HgCdTe}$ based on fundamental properties of the materials. Comparisons are made among QDIPs, QWIPs, and HgCdTe to emphasize fundamental differences.

\section{MODELS FOR DETECTOR TECHNOLOGY COMPARISON}

The ultimate performance of an IR detector is commonly referred to as the background limited performance (BLIP). This is the critical point where photogenerated carriers are equal to the noise in the detector, typically due to thermally generated carriers. Kinch has made an excellent comparison for fundamental limitations in IR detector material through analysis of the BLIP condition. ${ }^{21}$ This analysis allows one to evaluate a materials technology independent of device configuration and examine the BLIP condition where photogenerated carriers are greater than thermally generated carriers in the material, given by

$$
\eta \Phi \tau / t>n_{\text {th }},
$$

where $\eta$ is the absorption quantum efficiency, $\Phi$ is the photon flux, $\tau$ is the carrier lifetime, $t$ is the material thickness in the direction of the incident photon flux, and $n_{\text {th }}$ is the thermally generated carrier density. Using $\eta=\alpha t$, where $\alpha$ is the absorption coefficient in the material, we get the BLIP requirement $\Phi>n_{\text {th }} / \alpha \tau$ and obtain a normalized thermal generation rate $^{21}$

$$
G_{\mathrm{th}}=n_{\mathrm{th}} / \alpha \tau \text {. }
$$

This relationship provides a simplistic method of comparing fundamental differences between materials technologies for infrared detectors. 
The thermal generation rte for minority carrier detector devices is given by $G_{\text {th }}=n_{\min } / a \tau_{\min }$. For $n$-type $\mathrm{HgCdTe}$, minority carrier lifetime will be assumed to be limited by the Auger lifetime for intrinsic material and the thermal generation rate may be given by

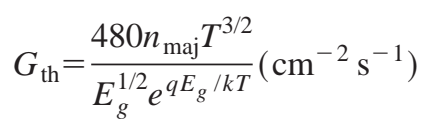

using well known material parameters for $\mathrm{HgCdTe}$, where $E_{g}$ is the band gap energy in $\mathrm{eV}$ and $n_{\text {maj }}$ is the majority carrier density in $\mathrm{cm}^{-3}$, which we will assume to be determined by the dopant density.

The normalized thermal generation rate for a QWIP may be given $b y^{21}$

$$
G_{\mathrm{th}}=2 \frac{n_{0}}{\alpha \tau} \frac{k T}{q E_{f}} \exp \left(q \frac{E_{f}-E_{2}}{k T}\right),
$$

where $E_{f}$ and $E_{2}$ are the Fermi energy and energy of the excited state transition in the quantum well, respectively. The equilibrium electron concentration in the ground state is given by $n_{0}$ and will be assumed to be similar to the doping level, typically near $10^{18} \mathrm{~cm}^{-3}$ for a QWIP. The absorption coefficient has a dependence on $n_{0}$, which has been found to be $\alpha=5 \times 10^{-15} n_{0} \mathrm{~cm}^{-1}$ for $\mathrm{GaAs}^{22}$ and will be used for this analysis. The factor of 2 in Eq. (4) is necessary for QWIPs due to the polarization selection rules which require the electric field of incident light to be parallel to the direction of quantum confinement, which results in a maximum absorption quantum efficiency of 0.5 for normal incidence operation. The generation rate is calculated assuming a net quantum well thickness equal to $1 / \alpha$ in order to maintain a fundamental comparison.

\section{FORMULATION FOR QDIPS}

The normalized thermal generation rate for a QDIP may be calculated using a similar technique. Here we will consider a planar array of quantum dots as illustrated in Fig. 1(a). A simple model will be assumed for the QD conduction band structure with two confined energy levels and the excited state transition coinciding with the barrier conduction band minimum, as shown in Fig. 1(b). General emphasis will be placed on quantum dots forming using the self-assembly technique where QDIPs have been demonstrated and some knowledge of the physical properties have been measured or calculated. A fill factor $F$ needs to be included for optical absorption in quantum dots. The fill factor is estimated as

$$
F=\frac{\sqrt[3]{V}}{s}
$$

where $V$ is the quantum dot volume and $s$ is the interdot spacing which equals $1 / D^{1 / 2}$. The optical absorption coefficient for intraband transitions is predicted to be larger for quantum dots than quantum wells due to increased localization of electrons in the quantum dots. An absorption coefficient of $>10^{4} \mathrm{~cm}^{-1}$ has been predicted for quantum dots. ${ }^{23,24}$ Quantum dots have a technological challenge in achieving a uniform quantum dot size. A significant degree of inhomogeneous broadening is observed in the electronic

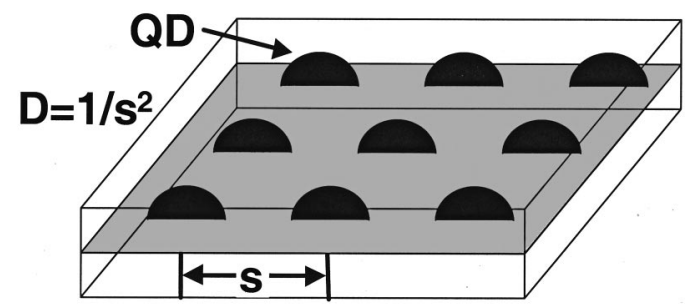

(a)

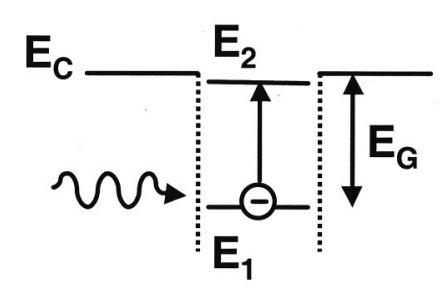

(b)

FIG. 1. Illustration of (a) the quantum dot array and (b) conduction band structure for the QDIP analysis.

spectra in quantum dot ensembles, and must be accounted for by proper evaluation of devices. For self-assembled quantum dots obtained through the Stranski-Krastanow growth mode, a Gaussian distribution has been observed for the electronic and optical spectra in quantum dot ensembles. The absorption spectra for an ensemble of quantum dots used in this analysis may be modeled using a Gaussian line shape and will be written as

$$
\alpha(E)=A \frac{n_{1}}{D} \frac{\sigma_{\mathrm{QD}}}{\sigma_{\text {ens }}} \exp \left(-\frac{\left(E-E_{G}\right)^{2}}{\sigma_{\text {ens }}^{2}}\right)\left[\mathrm{cm}^{-1}\right],
$$

where $A$ is the maximum absorption coefficient as obtained from calculations presented in the literature, $n_{1}$ is the areal density of electrons in the quantum dot ground state, $D$ is the quantum dot density, and $E_{G}$ is the energy of the optical transition between ground and excited states in the QDs $E_{G}$ $=E_{2}-E_{1}$. The expressions $\sigma_{\mathrm{QD}}$ and $\sigma_{\text {ens }}$ are the standard deviations in the Gaussian line shape [full width at half maximum is equal to $2 \sigma(2 \ln (2))^{1 / 2}$ ] for intraband absorption in a single quantum dot and for the distribution in energies for the quantum dot ensemble, respectively. This representation of the absorption coefficient accounts for the necessary presence of electrons in the quantum dot ground state for absorption to occur. The ratio $\sigma_{\mathrm{QD}} / \sigma_{\text {ens }}$ in Eq. (6) represents the decrease in maximum absorption coefficient due to inhomogeneous broadening of QD energy levels and the exponential term provides the Gaussian line shape. The modeling for intraband optical absorption in quantum dots given in Eq. (6) makes qualitative sense, where $A$ reflects the maximum absorption for QDs, and the terms $n_{1} / D$ and $\sigma_{\mathrm{QD}} / \sigma_{\text {ens }}$ describe a decrease in absorption due to the absence of available electrons in the QD ground state and inhomogeneous broadening, respectively.

The Fermi distribution is used to calculate the carrier densities. Quantum dot fabrication techniques such as selfassembled growth typically include a uniform sheet density of dopants to provide carriers for absorption. It is therefore 
necessary to include carriers and dopants outside of the quantum dot region. A Gaussian distribution may be used to reflect inhomogeneous broadening in ground and excited state levels in quantum dot ensembles, where electron densities for energy level $n$ are given by ${ }^{25}$

$$
n_{n}=\int \frac{g D}{\sqrt{\pi} \sigma} \exp \left(-\frac{\left(E-E_{n}\right)^{2}}{\sigma^{2}}\right) f\left(E_{n}\right) d E,
$$

where $g$ is the degeneracy factor for the energy level, $E_{n}$ is the mean energy, $\sigma$ is the standard deviation in energy for the Gaussian line shape, and $f(E)$ is the Fermi function. Pervious calculations for self-assembled InAs/GaAs quantum dots suggest a higher fourfold degeneracy for excited electron states. $^{23}$ We will assume a degeneracy of $g=2$ (two spin states) for the quantum dot ground state, and $g=8$ (fourfold degeneracy and two spin states each) for the quantum dot excited state. For calculations used in this analysis, the Gaussian line shape function describing carrier densities in QD ground and excited states has little effect on results provided $\sigma<E_{G}$, and may therefore be simplified to $n_{n}$ $=g D f\left(E_{n}\right)$. Using the charge neutrality condition, the twodimensional carrier densities may then be given by

$$
\begin{aligned}
N_{D}=n_{1}+n_{2}+n_{b}= & 2 D f\left(E_{1}\right)+8 D f\left(E_{2}\right) \\
& +\int_{0}^{\infty} g^{2 D}(E) f\left(E_{c}\right) d E,
\end{aligned}
$$

where $N_{D}$ is the sheet density dopant level, $D$ is the quantum dot density, $f(E)$ is the Fermi function, and $E_{1}$ and $E_{2}$ are the ground and excited state energies in the quantum dots, where $E_{2}-E_{1}=E_{g}$ is the energy of the quantum dot intraband transition. In this analysis we assumed, similar to the above QWIP, that $E_{2}$ coincides with the conduction band minimum of the barrier material $\left(E_{c}=E_{2}\right)$. The thermal carrier density is then given by $n_{2}+n_{b}$ and is converted to a three dimensional density by dividing by the net thickness $t$ of quantum dot layer. To maintain a fundamental comparison, we assume $t=1 / \alpha_{\max }$ to maintain a fundamental comparison. The normalized thermal generation rate may then be given by

$$
G=\frac{q \sigma D t}{A n_{1} F \tau}\left(n_{b}+n_{2}\right) .
$$

It should be noted that the carrier densities are dependent on temperature, dopant density, and quantum dot density, as indicated in Eq. (7).

\section{QDIP ANALYSIS}

Material parameters that will be used for QDIP calculations, unless otherwise noted, are listed in Table I. The value for $A$ is within the range reported in the literature. ${ }^{23,24}$ The volume and density of the QDs used in the calculations are representative of self-assembled InAs/GaAs quantum dots reported in the literature. ${ }^{12}$ The dopant concentration corresponds to 2 per QD. A value for $\tau$ has yet to be resolved for the QDIP device, although it is one of the important advantages and selling points of the QDIP over QWIPs. Phonon scattering is expected to be inhibited in quantum dots when
TABLE I. Parameters used for quantum dots in QDIP calculations, unless stated otherwise.

\begin{tabular}{cc}
\hline \hline$A$ & $5 \times 10^{4} \mathrm{~cm}^{-1}$ \\
$V$ & $5.3 \times 10^{-19} \mathrm{~cm}^{3}$ \\
$D$ & $5 \times 10^{10} \mathrm{~cm}^{-2}$ \\
$\tau$ & $1 \mathrm{~ns}$ \\
$N_{D}$ & $1 \times 10^{11} \mathrm{~cm}^{-2}$ \\
\hline \hline
\end{tabular}

the energy level separation between confined levels is larger than the thermal energy. Carrier relaxation times in quantum dots have been measured experimentally in $p-n$ junction laser devices to be $30-50$ ps. $^{26}$ These times are longer than the typical 1-10 ps measured for quantum wells. It is predicted that the carrier relaxation times in QDs are limited by electron-hole scattering, ${ }^{27}$ rather than phonon scattering. For QDIPs, the lifetime is expected to be even longer, greater than $1 \mathrm{~ns}$, for a majority carrier device such as the QDIP due to the absence of holes. In this article we assume $1 \mathrm{~ns}$ for the lifetime $\tau$.

QDIPs clearly have resemblance to QWIPs in general operation and device structure, however there are additional parameters unique to quantum dots that affect device performance. QDIPs essentially have more design parameters or "dials to turn," which are important to understand. QDIP performance has a distinct tradeoff between, and dependence on, quantum dot density and dopant concentration. Figure 2 shows the normalized dark current for a QDIP as a function of the $N_{D} / D$ ratio, where inhomogeneous broadening of the dot ensemble is neglected for clarity $\left(\sigma_{\mathrm{QD}} / \sigma_{\mathrm{ens}}=1\right)$. As one would expect, the optimal dopant density corresponds to twice the QD areal density (i.e., just enough dopants to fill the ground states of the QDs). The dark current increase at higher dopant densities as dopants outnumber the number of electron states available in the QD ground state. At reduced dopant densities, there are fewer electrons in the QD ground state for absorption. The reduced absorption results in the increased normalized dark current shown in Fig. 2 for low

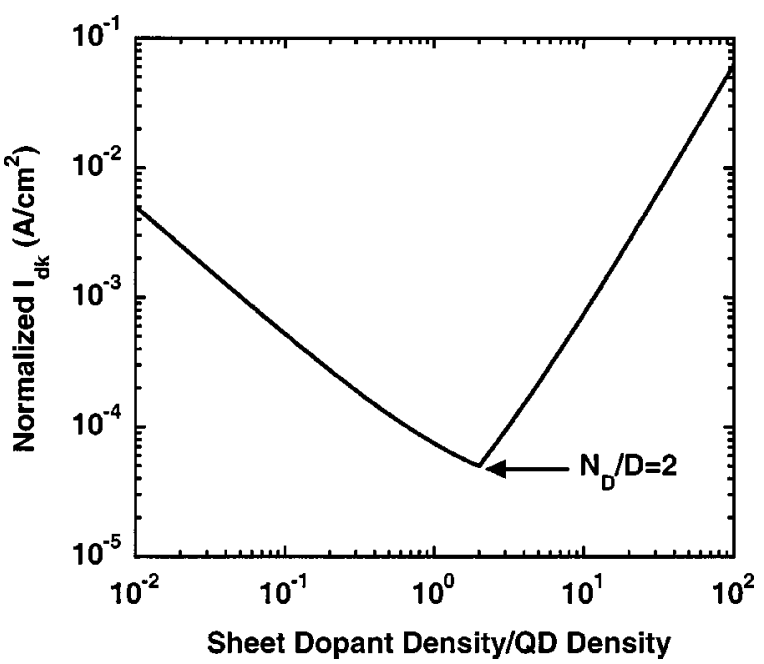

FIG. 2. Normalized dark current $(T=100 \mathrm{~K})$ for a QDIP vs the ratio of dopant density to quantum dot density for perfectly uniform QD ensemble $\left({ }_{\mathrm{QD}} / \sigma_{\mathrm{ens}}=1\right)$. 




FIG. 3. Quantum dot layer peak absorption equivalence compared to a single GaAs quantum well for varying QD uniformity.

dopant density, according to the dark current condition at BLIP used in this analysis. The result of increased normalized dark current at low dopant density may at first be counterintuitive, but it is clear that reduced absorption requires a larger optical material thickness for equivalent absorption.

It is useful to compare the absorption in QDIPs to QWIPs. Figure 3 shows the absorption equivalence of a layer of quantum dots versus dot density compared to a single 10 $\mathrm{nm}$ GaAs quantum well, for three cases of inhomogeneous broadening. For a perspective of these cases of inhomogeneous broadening, an assumed linewidth of $0.1 \mathrm{meV}$ translates to QD ensemble linewidths of 1 and $10 \mathrm{meV}$ for $\sigma_{\text {ens }} / \sigma_{\mathrm{QD}}$ ratios of 10 and 100 . The latter is similar to what has been demonstrated experimentally for self-assembled quantum dots. ${ }^{28}$ The larger absorption coefficient in quantum dots results in the ability to provide similar absorption at the peak energy to a quantum well for a perfectly uniform QD ensemble with density in the $10^{9}-10^{10} \mathrm{~cm}^{-2}$ range. For a single layer of QDs with $\sigma_{\text {ens }} / \sigma_{\mathrm{QD}}=100$, a QD density of $>10^{10} \mathrm{~cm}^{-2}$ is necessary to provide peak absorption equivalent to a single quantum well layer. It is clear that control over QD size uniformity is necessary to provide adequate absorption. Qualitatively, the inhomogeneous broadening in QDs results in a broadened spectral response with a weakened peak absorption and equivalent integrated absorption.

\section{HgCdTe, QWIP, AND QDIP COMPARISON}

A comparison of the normalized dark current for $\mathrm{HgCdTe}$, QWIP, and QDIP, as obtained in Eqs. (3), (4), and (8), is shown in Fig. 4 for a band gap energy of $E_{g}=0.124$ $\mathrm{eV}$ corresponding to a cutoff wavelength of $\lambda_{c} \sim 10 \mu \mathrm{m}$. It is clear from this analysis that the fundamental performance limitations of QWIPs are unlikely to rival HgCdTe. The performance of QDIPs, however, is predicted to rival and perhaps outperform $\mathrm{HgCdTe}$ for very uniform quantum dot arrays. Ryzhii ${ }^{29}$ has also theoretically evaluated the dark current in a QDIP and compared to a QWIP, and reached a similar prediction of a largely reduced dark current by a fac-

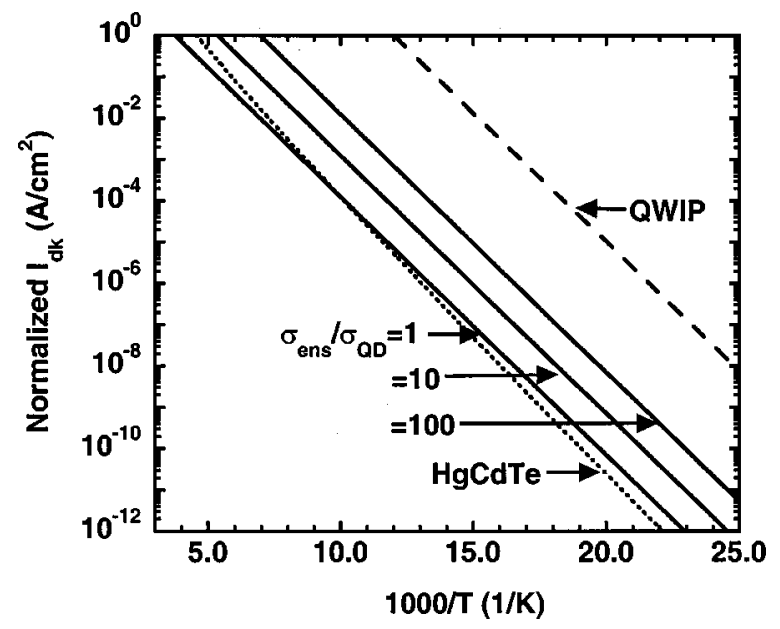

FIG. 4. Normalized dark current for HgCdTe, QWIP, and QDIP detectors as a function of temperature with a band gap energy of $10 \mu \mathrm{m}$.

tor of $0.1 \alpha_{\mathrm{QW}} / \alpha_{\mathrm{QD}}$. Ryzhii's analysis ${ }^{29}$ does not include the advantages of increased carrier lifetime in QDs, resulting in a higher predicted QDIP dark current than presented in this analysis.

A common figure of merit for detectors is the detectivity $D^{*}$, which reflects the signal/noise ratio independent of detector geometry and operation and is defined as

$$
D^{*}=\frac{\text { signal } \times \sqrt{\text { bandwidth }}}{\text { noise } \times \text { incidence } \times \sqrt{\text { area }}} .
$$

To compare these detector technologies, we will examine $D^{*}$ assuming that noise is limited by thermal generation. The noise current due to thermal generation is given by

$$
i_{n}=q \sqrt{2 G \times \text { area } \times \text { bandwidth }}
$$

and applying to Eq. (9), results in the following expression:

$$
D^{*}=\frac{\eta}{q h \nu \sqrt{2 G}}
$$

where $\eta$ is the external detector quantum efficiency and $h v$ is the energy of incident IR radiation. The detector quantum efficiency is assumed to be $\eta=1$ for $\mathrm{HgCdTe}$ and QDIPs (every photon absorbed, no reflection at surface) $\eta=0.5$ for QWIPs (cannot detect normal incidence radiation). The detectivity in Eq. (411) represents the upper limit for a given material then thermal noise is dominant over photon noise. The detectivity for $\mathrm{HgCdTe}$, QWIPs, and QDIPs are shown in Fig. 5 for a band gap energy of $E_{g}=0.124 \mathrm{eV}$. The detectivity of QDIPs is expected to outperform QWIPs by orders of magnitude and, in the case of highly uniform QD ensembles, rival, and potentially exceed that of $\mathrm{HgCdTe}$.

There have been several reports of QDIPs in the literature, but their performance has not been comparable to $\mathrm{HgCdTe}$ detectors. It is worth identifying reasons for QDIPs to perform far below expectations. Poor QDIP performance can generally be linked to two sources: nonoptimal band structure and nonuniformity in QD size. Quantum dot fabrication is intimately tied in to the final determination of the electronic band structure. In the analysis presented in this 




FIG. 5. Detectivity for $\mathrm{HgCdTe}$, QWIP, and QDIP detectors as a function of temperature with a band gap energy and incidence wavelength of $10 \mu \mathrm{m}$.

article, two QD electron energy levels are assumed, where the excited state coincides with the conduction band minimum of the barrier material. If the excited state transition lies far below the barrier conduction band, photocurrent is difficult to extract and may require operation at high bias. The reduced photocurrent will be reflected in low responsivity and detectivity. Quantum dots may also contain additional energy levels between the excited and ground state transitions. if these states are similar to the thermal temperature or permit phonon scattering between levels, carrier lifetime will be dramatically reduced and there will be a large increase in dark current and reduction in detectivity. Techniques such as the Stranski-Krastanow growth mode also result in a coupled two-dimensional "wetting layer," which causes some degradation of self-assembled quantum dots from the ideal state. Quantum dot fabrication will also affect absorption properties in the material. If lateral quantum confinement in QDs is small (i.e., they more closely resemble quantum wells), sensitivity to normal incidence radiation will be decreased, resulting in an increase in normalized dark current and reduced detectivity. One of the primary suspects for the current low performance of QDIPs is quantum dot size nonuniformity. Inhomogeneous broadening of QD energy levels is modeled in the analyses presented here and clearly illustrate how detector performance may be degraded by orders of magnitude for values of $\sigma_{\mathrm{ens}} / \sigma_{\mathrm{DQ}}=100$, which are indicative of the current state of QD fabrication technology. Nonuniformities in quantum dot size reduce the detector peak response and demand an increase in the number of QDs to counteract. The quantum efficiency of QDIPs measured experimentally has been generally low, $\sim 1 \%$, and has not come to equal that of QWIPs $(\sim 20 \%)$, suggesting that size uniformity needs to be improved and/or the ability to increase the number of quantum dots in a detector is needed. The reduced quantum efficiency for QDIPs in the comparison to QWIPs is consistent with the reduced QD peak absorption compared to quantum wells for the case of $\sigma_{\text {ens }} / \sigma_{\mathrm{QD}}=100$ shown in
Fig. 3. Reduced optical absorption in QDs due to size nonuniformity results in an increase in the normalized dark current and a reduction in detectivity.

\section{CONCLUSION}

The detection mechanism for intraband optical absorption in quantum dots has been described and modeled. The performance of QDIP detectors is expected to outperform QWIPs by several orders of magnitude when using measured or predicted parameters for self-assembled quantum dots. A direct comparison of limitations on dark current and detectivity in $\mathrm{HgCdTe}$, QWIP, and QDIP has been made. QDIPs are expected to have the potential to rival and exceed the performance of $\mathrm{HgCdTe}$ detectors if quantum dot arrays with high size uniformity may be achieved. Improvements in QD fabrication techniques to reduce inhomogeneous broadening are necessary to enable the competitiveness of QDIPs.

${ }^{1}$ P. R. Norton, Proc. SPIE 3379, 102 (1998).

${ }^{2}$ J. Bajaj, Proc. SPIE 3948, 42 (2000).

${ }^{3}$ M. B. Reine, Proc. SPIE 4028, 320 (2000).

${ }^{4}$ I. M. Baker and C. D. Maxey, J. Electron. Mater. 30, 682 (2001).

${ }^{5}$ J. B. Varesi et al., J. Electron. Mater. 30, 566 (2001).

${ }^{6}$ F. Fuchs, U. Weimar, E. Ahlswede, W. Pletschen, J. Schmitz, and M. Walther, Proc. SPIE 3287, 14 (1998).

${ }^{7}$ M. H. Young, D. H. Chow, A. T. Hunter, and R. H. Miles, Appl. Surf. Sci. 123/124, 395 (1998).

${ }^{8}$ H. Mohseni, M. Razeghi, G. J. Brown, and Y. S. Park, Appl. Phys. Lett. 78, 2107 (2001).

${ }^{9}$ S. D. Gunapala and K. Bandara, Thin Solid Films 21, 113 (1995), and references therein.

${ }^{10}$ S. D. Gunapala, S. V. Bandara, J. K. Liu, W. Hong, M. Sundaram, R. Carralejo, C. A. Chott, P. D. Maker, and R. E. Miller, Proc. SPIE 3061, 722 (1997).

${ }^{11}$ D. Pan, E. Towe, and S. Kennerly, Electron. Lett. 34, 1019 (1998).

${ }^{12}$ J. Phillips, K. Kamath, and P. Bhattacharya, Appl. Phys. Lett. 72, 2020 (1998).

${ }^{13}$ S. Kim, H. Mohseni, M. Erdtmann, E. Michel, C. Jelen, and M. Razeghi, Appl. Phys. Lett. 73, 963 (1998).

${ }^{14}$ Q. D. Zhuang, J. M. Li, H. X. Li, Y. P. Zeng, L. Pan, Y. H. Chen, and M. Y. Kong, Appl. Phys. Lett. 73, 3706 (1998).

${ }^{15}$ S. W. Lee, K. Hirakawa, and Y. Shimada, Appl. Phys. Lett. 75, 1428 (1999)

${ }^{16}$ J. Phillips, P. Bhattacharya, S. W. Kennerly, D. W. Beekman, and M. Dutta, IEEE J. Quantum Electron. 35, 936 (1999).

${ }^{17}$ H. C. Liu, M. Gao, J. McCaffrey, Z. R. Wasilewski, and S. Fafard, Appl. Phys. Lett. 78, 79 (2001).

${ }^{18}$ D. Leonard, M. Krishnamurthy, C. M. Reaves, S. P. Denbaars, and P. M. Petroff, Appl. Phys. Lett. 63, 3203 (1993).

${ }^{19}$ P. R. Berger, K. Chang, P. Bhattacharya, J. Singh, and K. K. Bajaj, Appl. Phys. Lett. 53, 684 (1988).

${ }^{20}$ H. Saito, K. Nishi, I. Ogura, S. Sugou, and Y. Sugimoto, Appl. Phys. Lett. 69, 3140 (1996).

${ }^{21}$ M. A. Kinch, J. Electron. Mater. 29, 809 (2000).

${ }^{22}$ B. F. Levine, C. G. Bethea, G. Hasnain, J. Walker, and R. J. Malik, Appl. Phys. Lett. 53, 296 (1988).

${ }^{23}$ H. Jiang and J. Singh, IEEE J. Quantum Electron. 34, 1188 (1998).

${ }^{24} \mathrm{~S}$. Li and J. Xia, Phys. Rev. B 55, 15434 (1997).

${ }^{25}$ P. N. Brounkov, A. Polimeni, S. T. Stoddart, M. Henini, L. Eaves, P. C. Main, A. R. Kovsh, Yu. G. Musikhin, and S. G. Konnikov, Appl. Phys. Lett. 73, 1092 (1998).

${ }^{26}$ D. Klotzkin, K. Kamath, and P. Bhattacharya, IEEE Photonics Technol. Lett. 9, 1301 (1997).

${ }^{27}$ I. Vurgaftman, Y. Lam, and J. Singh, Phys. Rev. B 50, 14309 (1994).

${ }^{28}$ S. Krishna, J. Sabarinathan, K. Linder, P. Bhattacharya, B. Lita, and R. S. Goldman, J. Vac. Sci. Technol. B 18, 1502 (2000).

${ }^{29}$ V. Ryzhii, Semicond. Sci. Technol. 11, 759 (1996). 